\title{
TOPICS IN STATISTICAL PHYSICS INVOLVING BRAIDS
}

\author{
J. McCABE \\ Laboratoire de Physique Théorique, ENSLAPP, ENS Lyon \\ 46 allée d'Italie, F-69364 Lyon Cedex 0\%, France \\ Present address: 2525 South Voss Rd, \#206, Houston, TX 77057, U.S.A. \\ E-mail: mccabe@hypercon.com \\ T. WYDRO \\ Laboratoire de Physique des Liquides et des Interfaces, Institut de Physique \\ Université de Metz, 1, bd Arago, F-57000 Metz, France \\ E-mail: wydro@lpli.univ-metz.fr
}

\begin{abstract}
We review the appearance of the braid group in statistical physics. In particular, we explain its relevance to the anyon model of fractional statistics and conformal field theory.

This short contribution reviews two theories in statistical physics: conformal field theory and the anyon model. Our research has concerned studies of the duality equation in conformal theories and the energy spectrum in the anyon model. While our interests have concentrated on physical issues, both of these subjects have intimate connections with the braid group. This article will expand our oral presentation to discuss those mathematical connections. We hope that a review format, where one of the principle goals is to clarify the origin of braid statistics, will aid the present audience and encourage it to address the unanswered mathematical issues presented.

The remainder of this contribution is organized as follows. The first section of the article presents some generalities on braid group statistics that are relevant to both theories. The second section describes the anyon model. We have chosen to start with it, because it is inherently simple and does not require discussions of advanced topics in physics. The third section considers correlation functions in conformal field theory. The physical origins of this theory are briefly presented. Then, we develop in some detail the machinery that has led to the construction of correlation functions. The braid group only occurs in the tools of this construction, i.e. the conformal blocks. We end by illustrating the connection with the duality equation.
\end{abstract}

1991 Mathematics Subject Classification: 82-02, 82Bxx, 81-02, 81Rxx.

The paper is in final form and no version of it will be published elsewhere. 
The presentation avoids technical developments in some places. We have tried to indicate where. We have attempted to retain enough details so that the logic is not lost. A reader who wants to have more information should look at one of the longer reviews: [4] on anyons, [8] on conformal field theory, and [14] on the relation between conformal theory and braids and further connections to knots.

1. Braid group statistics. We will be interested by generalized forms of statistics in systems of identical particles. In quantum mechanics, the behavior of $N$ identical particles is described by the wave function, a complex function of the particle coordinates $\vec{x}_{j}\left(\vec{x}_{j} \epsilon \mathbf{R}^{d}, j=1,2 \ldots N\right)$. For identical particles, one might expect that the wave function is invariant under all permutations, $P$, of the particle names, i.e. transformations of the form $\vec{x} \rightarrow \vec{x}_{P(j)}$. The real situation is more complicated, because wave functions differing only by a constant phase describe the same physical system. Thus, the wave function can be in a nontrivial one dimensional representation of the permutation group. There are two such representations, the symmetric and anti-symmetric representations. They correspond to multiplication of the wave function by +1 or -1 under a transposition of the coordinates of any two particles:

$$
\Psi\left(\vec{x}_{1}, \ldots, \vec{x}_{i}, \ldots, \vec{x}_{j}, \ldots\right)= \pm \Psi\left(\vec{x}_{1}, \ldots, \vec{x}_{j}, \ldots, \vec{x}_{i}, \ldots\right) .
$$

This gives the two well-known "statistics" for identical particles. Symmetric wave functions describe Bose particles, and anti-symmetric wave functions describe Fermi particles. Though all physical theories of $N$ identical particles fall into one of these two classes, we will find it profitable to try to find generalizations.

In the late 1970's, Leinaas and Myrheim described a different way of defining the statistics of the wave function [1]. The basic idea is to replace permutations of the particle coordinates with continuous transformations. First, we will study the situation for the two particle system. It is convenient to introduce the center of mass, $\vec{Z}=\vec{x}_{1}+\vec{x}_{2}$, and relative, $\vec{X}=\vec{x}_{1}-\vec{x}_{2}$, coordinates. A transposition of the two particles only acts on the relative coordinate, i.e. $\vec{X} \rightarrow-\vec{X}$. To generalize permutations to continuous transformations, we introduce the vector functions $\vec{F}(t)$ satisfying $\vec{F}(0)=\vec{X}$ and $\vec{F}(1)=-\vec{X}, t \in[0,1]$. In generalized statistics the sign in (1) is replaced by a phase. The phase change of the wave function as $\vec{X}$ is moved along the path $\vec{F}(t)$ defines the statistics.

$$
\Psi(\vec{Z}, \vec{X})=e^{i \alpha(\vec{F})} \Psi(\vec{Z},-\vec{X})
$$

The statistics should not depend on the detailed form of the path. Thus, we require that the phase function $e^{i \alpha(\vec{F})}$ only depend on the equivalence class of the path $\vec{F}(t)$. Finally, the phase change of a function is additive as its coordinate is transported first along one path and then along a second one. A consistent definition of statistics requires that the defining phase in (2) satisfy a similar additivity property on paths that can be decomposed into several paths.

There is a constraint on $e^{i \alpha(\vec{F})}$ coming from the dimension, $d$, of physical space. To see this, we make the transport along the path defined by: $\vec{F}^{\prime}(t)=\vec{F}(2 t)$ for $0 \leq t \leq \frac{1}{2}$ and $\vec{F}^{\prime}(t)=-\vec{F}(-1+2 t)$ for $\frac{1}{2} \leq t \leq 1$. Along this closed path, the wave function gets 
a total phase change:

$$
e^{i \alpha\left(\vec{F}^{\prime}\right)}=e^{2 i \alpha(\vec{F})}
$$

Since $\vec{F}^{\prime}(t)$ is a closed path and the phase is independent of its detailed form, the first homotopy group gives a classification of the possible phases $e^{2 i \alpha(\vec{F})}$. If this is to result in a generalization of normal statistics, the phase in (3) must not be identically zero. For this to be possible, there must be nontrivial homotopy classes.

The individual particle coordinates $\vec{x}_{1}$ and $\vec{x}_{2}$ are points in $\mathbf{R}^{d}$. But, physical considerations ${ }^{1}$ impose a constraint on the total configuration space: $\vec{x}_{1} \neq \vec{x}_{2}$. Thus, the relative coordinate $\vec{X}$ is a point in $\mathbf{R}^{d} \backslash\{\overrightarrow{0}\}$. On $\mathbf{R}^{d} \backslash\{\overrightarrow{0}\}$, the first homotopy group is trivial for $d=3,4, \ldots$. This implies that $e^{2 i \alpha(\vec{F})}=1$. Thus, for all open paths $e^{i \alpha(\vec{F})}= \pm 1$, and the only possibilities are Bose or Fermi statistics. The case of $d=2$ is special. The first homotopy group on $\mathbf{R}^{2} \backslash\{\overrightarrow{0}\}$ is $\mathbf{Z}$. Thus, there is no constraint on the statistics parameter $e^{2 i \alpha(\vec{F})}$. We have found that generalized statistics are only possible in two dimensions.

In two dimensions, the $N$-particle configuration space becomes $\mathbf{Q}=\left(\mathbf{R}^{2}\right)^{N} \backslash \mathbf{D}$. Again, D is the subspace of $\left(\mathbf{R}^{2}\right)^{N}$ where $\vec{x}_{j}=\vec{x}_{j}$ for any two particles $i$ and $j$. It must be removed for the same physical reasons invoked above. The transformation of the $N$ particle wave function can be defined by introducing a set of $N$ nonintersecting paths on $\left(\mathbf{R}^{2}\right)^{N}$, i.e. $\vec{F}_{j}(t)$ with $j=1,2, \ldots N$. The $\vec{F}_{j}(t)$ form a $N$-braid if $\vec{F}_{j}(0)=\vec{x}_{j}$ and $\vec{F}_{j}(1)=\vec{x}_{P(j)}$. The statistics is defined by the transport of the wave function's coordinates along the braid.

$$
\Psi\left(\vec{x}_{1}, \ldots, \vec{x}_{j}, \ldots, \vec{x}_{N}\right)=e^{i \alpha(\vec{F})} \Psi\left(\vec{F}_{1}(1), \ldots, \vec{F}_{j}(1), \ldots, \vec{F}_{N}(1)\right)
$$

Again, the phase change of the wave function only depends on the particular braid along which the coordinates are transported. Furthermore, a consistent definition of statistics in $(4)$, requires that the phase $e^{i \alpha(\vec{F})}$ be additive on braids that can be decomposed into successively traversed braids. Different choices for the phase function will lead to different generalized statistics.

Since the phase function $e^{i \alpha(\vec{F})}$ is additive on braids that are successively traversed, the wave function is in a one dimensional representation of the braid group. This leads to constraints on the phases. All elements of the braid group can be made from fundamental interchanges, $\sigma_{j}$, i.e. the twists:

$$
\left(\vec{x}_{1}, \ldots, \vec{x}_{j}, \vec{x}_{j+1}, \ldots, \vec{x}_{N}\right) \rightarrow\left(\vec{x}_{1}, \ldots, \vec{x}_{j+1}, \vec{x}_{j}, \ldots, \vec{x}_{N}\right) .
$$

They satisfy the following relations [2]:

$$
\sigma_{i} \sigma_{j}=\sigma_{j} \sigma_{i} \text { for }|i-j| \geq 2 \text { and } \sigma_{i+1} \sigma_{i} \sigma_{i+1}=\sigma_{i} \sigma_{i+1} \sigma_{i}
$$

These equations constrain the phases defining the one dimensional representation to satisfy $e^{i \alpha\left(\sigma_{i}\right)}=e^{i \alpha\left(\sigma_{j}\right)}$ for all $i$ and $j$. The single remaining value of $e^{i \alpha(\sigma)}$ will define the generalized statistics of the particles.

\footnotetext{
${ }^{1}$ There is always a short distance repulsion between particles that forbids any two from occupying the same point.
} 
We close this section with one remark. Since generalized statistics only occurs in 2 dimensions, we can use complex coordinates to designate the position of the particles. In those coordinates, generalized statistics will require that wave functions have cuts at the position of each particle.

2. Anyon model of fractional statistics. The anyon model is a quantum mechanical theory of identical particles which realizes braid group statistics. The quantum properties of any system of $N$ particles come from solutions of an eigenvalue equation, $H \Psi_{[a]}\left(\vec{x}_{1}, \ldots, \vec{x}_{N}\right)=E_{[a]} \Psi_{[a]}\left(\vec{x}_{1}, \ldots, \vec{x}_{N}\right)$. These equations determine both the stationary state wave functions $\Psi_{[a]}\left(\vec{x}_{1}, \ldots, \vec{x}_{N}\right)$ and the energies $E_{[a]}$. For a nonrelativistic free particle system the operator $H$ has the form $H=\frac{1}{2 M} \sum_{j=1}^{N}\left(i \vec{\partial}_{j}\right)^{2}, M$ is the particle mass. The eigefunctions are well-known for the free Bose and Fermi cases. They are the plane wave states ${ }^{2}$.

Anyons are nonrelativistic Bose particles having a self-interaction. The interaction between the anyon at $\vec{x}_{j}$ and the other $N-1$ anyons can be written through a gauge field $\vec{A}\left(\vec{x}_{j}\right)$ and an anyon charge $\alpha$. Thus, for $N$ anyons, the operator $H$ is:

$$
H=\frac{1}{2 M} \sum_{j=1}^{N}\left(i \vec{\partial}_{j}-\alpha \vec{A}\left(\vec{x}_{j}\right)\right)^{2} .
$$

The field $\vec{A}\left(\vec{x}_{j}\right)$ is a normal electromagnetic potential, but its form is very special.

$$
\vec{A}\left(\vec{x}_{j}\right)=\sum_{i \neq j} \frac{\vec{n} \times\left(\vec{x}_{j}-\vec{x}_{i}\right)}{\left|\vec{x}_{i}-\vec{x}_{j}\right|^{2}} .
$$

Here the unit vector $\vec{n}$ is perpendicular to the plane on which the particles move. We have written the form of $\vec{A}\left(\vec{x}_{j}\right)$ for anyons moving on $\mathbf{R}^{2}$. Its form will be somewhat different if one introduces periodic boundary conditions to discretize the spectrum of $H$, i.e. see footnote 2 .

The field potential will tell us something about the character of anyons. One sees this by calculating the local magnetic field, $B$, felt by the particle at $\vec{x}_{j}$. Using the definition of the magnetic field, $B(\vec{x})=\vec{\nabla} \times \vec{A}(\vec{x})$, one finds that ${ }^{3}$ :

$$
B\left(\vec{x}_{j}\right)=2 \pi \sum_{i \neq j} \delta^{2}\left(\vec{x}_{j}-\vec{x}_{i}\right) .
$$

Thus, the magnetic field is a sum of point sources localized at the position $\vec{x}_{i}$ of each anyon. The anyon gas is a collection of point magnetic fluxes of intensity $2 \pi$ that interact due to the fact that they also carry a charge $\alpha$, see fig. 1 .

The anyons that we have described are normal Bose particles, and their wave function is symmetric under the exchange of any two. As such, anyons are interacting. It is also

\footnotetext{
${ }^{2}$ To solve these equations one introduces boundary conditions that make the operator $H$ hermitian. One choice is periodic conditions: $\Psi_{[a]}\left(\vec{x}_{1}, \ldots, \vec{x}_{j}, \ldots\right)=\Psi_{[a]}\left(\vec{x}_{1}, \ldots, \vec{x}_{j}+\vec{L}, \ldots\right)$ with $\vec{L} \in\{(0, L),(L, 0)\}$. Bose, $(+1)^{P}$, and Fermi, $(-1)^{P}$, solutions are: $\Psi_{\left[\vec{k}_{1}, \ldots, \vec{k}_{N}\right]}=$ $\sum_{P}( \pm 1)^{P} \exp \left(i \sum_{j} \vec{k}_{j} \cdot \vec{x}_{P(j)}\right), E_{\left[\vec{k}_{1}, \ldots, \vec{k}_{N}\right]}=\frac{1}{2 M} \sum_{j} k_{j}^{2}, \vec{k}_{j} \in 2 \pi(n, m) / L$.

${ }^{3}$ In this calculation, we use: $\vec{\nabla} \cdot\left(\vec{x} / x^{2}\right)=2 \pi \delta^{2}(\vec{x})$ with $\delta^{2}(\vec{x})$ the $2 D$ Dirac delta function.
} 


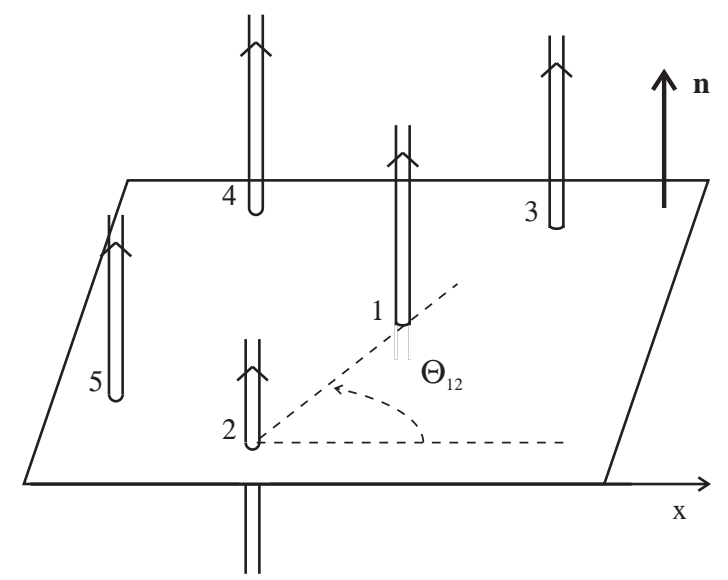

Fig. 1. Picture of a system of 5 anyons as magnetic flux tubes

possible to express the anyon model in an equivalent form which involves "free" particles with braid statistics. The "free" form occurs, because the vector potential of (7) is the gradient of a function. We will show this in the two particle case. In the coordinates $(x, y)=\left(x_{1}-x_{2}, y_{1}-y_{2}\right)$, the vector potential is $\left(A_{x}, A_{y}\right)=\left(-y / r^{2}, x / r^{2}\right)$. In radial coordinates, $(x, y)=(r \cos \Theta, r \sin \Theta)$, this can be rewritten as $\left(A_{x}, A_{y}\right)=\left(\partial_{x} \Theta, \partial_{y} \Theta\right)$. $\Theta$ is the angle that the vector $(x, y)$ makes with the $x$-axis. Thus, $\vec{A}$ is the gradient of a function, but this function, $\Theta$, is multi-valued on the plane. Usually, $\vec{A}$ 's that are gradients can be totally removed by a gauge transformation. Here, the gauge transformation will be multi-valued like $\Theta$; it will change the statistics. Gauge transformations are unitary transformations that act on both $\vec{A}$ and the wave function. They leave the energy eigenvalues, $E_{[a]}$, invariant. It is easily seen that the eigenvalue equation,

$$
H \Psi_{[a]}\left(\vec{x}_{1}, \ldots, \vec{x}_{N}\right)=E_{[a]} \Psi_{[a]}\left(\vec{x}_{1}, \ldots, \vec{x}_{N}\right),
$$

is invariant under any local transformation of the form:

$$
\vec{A}^{\prime}\left(\vec{x}_{j}\right)=\vec{A}\left(\vec{x}_{j}\right)-\frac{1}{\alpha} \vec{\partial}_{j} \Lambda\left(\vec{x}_{j}\right) \text { and } \Psi_{[a]}^{\prime}=e^{i \Lambda\left(\vec{x}_{j}\right)} \Psi_{[a]}\left(\vec{x}_{1}, \ldots, \vec{x}_{N}\right)
$$

Since $\vec{A}(x, y)=\vec{\partial} \Theta(x, y)$, the choice $\Lambda(x, y)=\alpha \Theta(x, y)$ is very interesting in the two particle case. From (9), we see that this gauge transformation will make $\vec{A}$ vanish. For this choice, the primed form of the anyon model is a "free" theory, i.e. $H^{\prime} \Psi^{\prime}\left(\vec{x}_{1}, \vec{x}_{2}\right)=$ $(-1 / 2 M)\left(\vec{\partial}_{1}^{2}+\vec{\partial}_{2}^{2}\right) \Psi^{\prime}\left(\vec{x}_{1}, \vec{x}_{2}\right)$. Now, we will see that the price for rewriting the anyon model as a free theory is braid statistics. To find the statistics of the new wave function, we recall that the original wave function was bosonic: $\Psi\left(\vec{x}_{1}, \vec{x}_{2}\right)=+\Psi\left(\vec{x}_{2}, \vec{x}_{1}\right)$. The transformation from $\Psi$ to $\Psi^{\prime}$ involved a multi-valued phase factor $e^{i \Lambda(x, y)}=e^{i \alpha \Theta(x, y)}$. Thus, the primed wave function transforms differently than the unprimed one (Bose) under the exchange of the two particles. It satisfies:

$$
\Psi^{\prime}\left(\vec{x}_{1}, \vec{x}_{2}\right)=e^{i \alpha[\Theta(\vec{F}(1))-\Theta(\vec{F}(0))]} \Psi^{\prime}\left(\vec{x}_{2}, \vec{x}_{1}\right) \equiv e^{ \pm i \alpha \pi} \Psi^{\prime}\left(\vec{x}_{2}, \vec{x}_{1}\right)
$$

In this equation, the angle $\Theta(\vec{F}(1))-\Theta(\vec{F}(0))$ is a function of the 2-braid, $\vec{F}(t)$, that defines the exchange of $\vec{x}_{1}$ and $\vec{x}_{2}$. The last equality in (10) is correct for the $\sigma$ - 
twists of (5). The \pm sign ambiguity is determined by whether $\vec{x}_{1}$ is braided around $\vec{x}_{2}$ in a clockwise or anti-clockwise sense. The primed "free" wave functions form a one dimensional representation of braid group except when $\alpha=0$ (Bose statistics) or $\alpha=1$ (Fermi statistics). Varying $\alpha$ from 0 to 1 smoothly changes the model from free Bose particles to free Fermi particles.

In the primed theory, the energies $2 M E_{[a]}$ are given by the eigenvalues of the free $4 D$ Laplacian, $-\vec{\partial}_{1}^{2}-\vec{\partial}_{2}^{2}$. The eigenfunctions are representations of the 2-braid group with statistics $e^{i \alpha(\sigma)}=e^{i \alpha \pi}$, see sec. 1 . One can use circular box boundary conditions, $\left.\Psi^{\prime}\right|_{\left|\vec{x}_{1}-\vec{x}_{2}\right|=R}=0$, with $R$ the radius of the box, to define the eigenfunctions of the Laplacian. With these boundary conditions, one finds the following eigenfunctions [1][3]:

$$
\Psi_{\vec{p}}^{\prime}(r, \Theta)=e^{i(n+\alpha) \Theta} J_{n+\alpha}(p r) \text { where } E=p^{2} / M
$$

The boundary conditions fix $p R$ to be the zeros of the Bessel function: $J_{n+\alpha}(p R)=0$. The doublets $[n, p]$, with $n$ integer, are the quantum numbers that label the states and their energies. The dependence of the solutions on the center of mass $\vec{Z}=\vec{x}_{1}+\vec{x}_{2}$ is trivial and has not been indicated. These explicit solutions for the "free" version of the anyon theory's wave functions transform, as shown in (10), under the braid group.

The $N$-anyon energy eigenstates can also be obtained by solving the eigenvalue problem of a free Laplacian. The eigenfunctions form a one dimensional representation of the $N$-braid group. To see this, we notice that the vector potential of $N$-anyons can also be written as a pure gradient, i.e. $\vec{A}\left(\vec{x}_{j}\right)=\vec{\partial}_{j}\left(\sum_{k<q} \Theta_{k q}\right)$ with $k, q=1, \ldots, N$. $\Theta_{k q}$ is the angle that the vector $\vec{x}_{k}-\vec{x}_{q}$ makes with the $x$-axis. This "pure gauge" form is easily verified from the 2-particle case. It tells us that there also exists a gauge transformation (9), in the $N$-particle case, that transforms the Hamiltonian to a free form, i.e. $H^{\prime}=(1 / 2 M) \sum_{j=1}^{N}\left(-\vec{\partial}_{j}^{2}\right)$. The gauge transformation to the "free" primed theory has the parameter $\Lambda\left(\vec{x}_{1}, \ldots, \vec{x}_{N}\right)=\alpha \sum_{k<q} \Theta_{k q}\left(\vec{x}_{k}-\vec{x}_{q}\right)$. One can easily see that the primed eigenfunctions, $\Psi^{\prime}\left(\vec{x}_{1}, \ldots, \vec{x}_{N}\right)$, form $1 D$ representations of the $N$-braid group (5) with statistics $e^{i \alpha(\sigma)}=e^{i \alpha \pi}$. Clearly, this "free" theory smoothly interpolates between the free Bose model at $\alpha=0$ and the free Fermi model at $\alpha=1$ exactly as in the 2-anyon case.

The energy eigenvalues for 3 and 4 anyons have been studied, but the results are very incomplete [5]. The connection with free Laplace eigenfunctions having braid group statistics has only been exploited in the 2-anyon case. A knowledge of the eigenvalues of the $N$-particle case would allow a calculation of the thermodynamic properties of the anyon gas at high densities. Like the perfect Bose and Fermi gas, the anyon gas is "perfect" or "free." It is a perfect gas with arbitrary statistics. The quantum mechanics of all three gases require finding the eigenvalues of the $2 N$-dimensional Laplacian. The Bose and Fermi cases were, however, simpler as the wave functions are representations of the permutation group - the so-called plane wave solutions (footnote 2). The anyon gas must have an energy spectrum which is quite different from either of these two limiting cases. Widely believed approximate results say that at high density and for $\alpha=1 / s$, with $s \in \mathbf{Z}$, gaps appear in the energy spectrum [6]. This does not happen in either the Bose or Fermi gases. An understanding of plane wave solutions that obey braid group statistics would have to explain these results. 
3. Correlation functions in conformal field theory and braids. We introduce conformal theories by way of an example which is one of the successes of statistical physics, the Ising model. It is the simplest of a family of models with short range interactions. It has only one dynamical field, the spin $S_{j}$, defined at each site $j$ of a regular lattice $\Gamma$. The spin takes two values \pm 1 . The interaction between spins is determined by the form of the energy $E=-J \sum_{<i, j>} S_{i} S_{j}$. The interaction has strength $J$ and is only nonzero between spins at nearest neighbor sites $\langle i, j\rangle$. Even in this simple model, the number of microscopic states is enormous, equal to $2^{N}$ where $N$ is the number of lattice sites. In statistical physics the major goal is to find a simple macroscopic description from this microscopic complexity.

The macroscopic comportment of the Ising model is typical of many systems. It has two phases - a high temperature phase where the macroscopic average spin vanishes: $\sum_{j \in \Gamma}<<S_{j}>>=0$, the disordered phase ${ }^{4}$, and a low temperature phase with a nonzero macroscopic spin $\sum_{j \in \Gamma}<<S_{j}>>\neq 0$, the ordered phase. The transition from zero to nonzero average spin occurs at a precise temperature $T_{c}$. Near the transition point, the model is characterized by two macroscopic dimensionful quantities $-\sum_{j \in \Gamma}<<S_{j}>>$ and $\left(T-T_{c}\right)$. Nevertheless, exactly at $T_{c}$ exactly $\sum_{j \in \Gamma}<<S_{j}>>=0$ in the Ising model. Thus, there are no "dimensionful" macroscopic quantities that characterize the model at $T_{c}$. Transitions involving no dimensionful parameters are called second order phase transitions. For this type of transition, physical properties show distinctly different behavior at $T_{c}$, the critical point, and away from $T_{c}$. As an example, one can look at correlation functions for two spins. Away from $T_{c}$, they depend on a length scale $\xi\left(T-T_{c}\right)$, falling off exponentially with the distance between the spins, i.e. $\left\langle<S_{\overrightarrow{0}} S_{\vec{r}}>>\simeq e^{-r / \xi}\right.$. At $T_{c}$, there is no dimensionful scale, i.e. at $T_{c} \xi(0) \rightarrow \infty$, and the correlation exhibits power law behavior, i.e. $\left\langle<S_{\overrightarrow{0}} S_{\vec{r}}>>\simeq r^{-4 \Delta_{S}}\right.$. There is direct experimental evidence of this fundamental distinction. At critical point, the no-scale correlation function indicates the presence of fluctuations at all length scales, this greatly enhances light scattering. This effect has been observed, and it is called critical opalescence.

The scale invariance of critical points led to several profound ideas on the relationship between the macroscopic descriptions and the microscopic statistical models - universality, renormalization group and conformal field theory. Universality says that many details of the microscopic model are washed out at $T_{c}$. Many different microscopic models are described by the same "effective" critical theory. This is the reason that a simple theory like the Ising model can describe real physical systems. The renormalization group was one of the most important tools in the calculation of macroscopic quantities from the underlying models. Its most important predictions were exponents like $\Delta_{S}$ defining the 2-spin correlation function. Conformal field theories exploit the group theory of local scale transformations to obtain the "effective" macroscopic theory. For $2 D$ systems, they have given an explicit implementation of universality - an almost "complete" classification of macroscopic critical theories. This led to two types of predictions - the values of exponents like $\Delta_{S}$ and the form of correlation functions involving more than two fields.

\footnotetext{
${ }^{4}<<A>>$ designates the thermal average of any object, i.e. $<<A>>\equiv \sum_{a \in \text { states }} A e^{-E_{a} / k T}$.
} 
In 1970, Polyakov postulated that the scale invariance of a critical point implied that the macroscopic theory had conformal symmetry [7]. The finite conformal group contains rotation, scale, translation, and special conformal transformations. The infinitesimal transformations with parameters $M_{i j}=-M_{j i}, \Lambda, \vec{a}$ and $\vec{b}$ have the form:

$$
x_{k}^{\prime}=x_{k}+\sum_{j=1}^{d} M_{k j} x_{j}, x_{k}^{\prime}=x_{k}+\Lambda x_{k}, x_{k}^{\prime}=x_{k}+a_{k}, x_{k}^{\prime}=x_{k}+x^{2} b_{k}-2 \vec{b} \cdot \vec{x} x_{k}
$$

Polyakov showed that all correlation functions of local fields were strongly constrained by conformal invariance. Using this symmetry, he was able to find the form of the two and three point correlations up to some exponents and constants, $C_{i j k}$, the structure constants $^{5}$.

$$
\begin{gathered}
<<\phi_{i}\left(\vec{x}_{1}\right) \phi_{j}\left(\vec{x}_{2}\right)>>=\frac{N_{i} \delta_{i, j}}{\left|\vec{x}_{1}-\vec{x}_{2}\right|^{4 \Delta_{i}}} \\
<<\phi_{i}\left(\vec{x}_{1}\right) \phi_{j}\left(\vec{x}_{2}\right) \phi_{k}\left(\vec{x}_{3}\right)>>= \\
\frac{\sqrt{N_{i} N_{j} N_{k}} C_{i j k}}{\left|\vec{x}_{1}-\vec{x}_{2}\right|^{2\left(\Delta_{i}+\Delta_{j}-\Delta_{k}\right)}\left|\vec{x}_{2}-\vec{x}_{3}\right|^{2\left(\Delta_{j}+\Delta_{k}-\Delta_{i}\right)}\left|\vec{x}_{3}-\vec{x}_{1}\right|^{2\left(\Delta_{k}+\Delta_{i}-\Delta_{j}\right)}}
\end{gathered}
$$

The fields $\phi_{j}(\vec{x})$ are the primary fields of the model. In the Ising model, they are $S_{j}$ and the local energy density $E_{j}=-J \sum_{\vec{k}=\vec{j}+\vec{n}} S_{\vec{k}} S_{\vec{j}} \cdot{ }^{6}$ The constants $C_{i j k}$ and exponents are universal objects defined uniquely by the critical theory. This has been shown constructively in 2 dimensions, but it took 14 years to formulate the proof.

The proof is based on an extension of conformal symmetry to an infinite group [8]. This extended symmetry is the foundation of modern conformal field theory. Further developments based on the infinite conformal symmetry group led to the calculation of the exponents [9] and structure constants [8][10] of many models (ex. $C_{S S E}=1 / 2, \Delta_{E}=$ $1 / 2, \Delta_{S}=1 / 16$ in the Ising model). These predictions have been tested in numerical simulations [11].

In 2 dimensions, one can show that the invariance of a field theory under the finite group guarantees invariance under a much larger symmetry group [8]. The full symmetry group is easier to write if we use complex coordinates $(z, \bar{z})$ to describe the position of local fields like the spin, $S(z, \bar{z})$, and the energy density, $E(z, \bar{z})$. The infinitesimal transformations of the full symmetry group are all analytic or anti-analytic transformations on $(z, \bar{z})$.

$$
z^{\prime}=z+\sum_{n \in Z} a_{n} z^{n} \text { and } \bar{z}^{\prime}=\bar{z}+\sum_{n \in Z} \bar{a}_{n} \bar{z}^{n}
$$

Here, $a_{n}$ and $\bar{a}_{n}$ are the independent parameters of the transformation and are defined $^{7}$ for negative and positive integers $n$. On functions of $(z, \bar{z})$, these transforma-

\footnotetext{
${ }^{5}$ At $T_{c}$, we replace $<<A>>\rightarrow<0|A| 0>$. Under the transformation of (12), the critical ground state $\mid 0>$ is invariant, but fields transform: $\phi_{j}^{\prime}(\vec{x})=\left|\partial x_{k}^{\prime} / \partial x_{l}\right|^{\Delta_{j}} \phi\left(\vec{x}^{\prime}\right)$. The $N_{j}$ 's are dropped from here on. They are only relevant to experimental measurements.

${ }^{6} \vec{n}$ are the primitive lattice vectors generating $\Gamma$.

${ }^{7}$ For physical reasons, conformal field theories are actually defined on the plane without origin. Thus, the transformations can be singular at $z=0$.
} 
tions are generated by $L_{n}=-z^{n+1} \partial_{z}$ and $\bar{L}_{n}=-\bar{z}^{n+1} \partial_{\bar{z}}$. Their commutators satisfy the Virasoro algebra, actually two copies one from the $L_{n}$ 's and one from the $\bar{L}_{n}$ 's.

$$
\left[L_{n}, L_{m}\right]=(n-m) L_{n+m}+\frac{c}{12} n\left(n^{2}-1\right) \delta_{n,-m}
$$

On normal functions, this algebra does not contain the second term on the right hand side, i.e. $c=0$. In conformal field theory, one constructs a representation of this algebra on physical fields,$\phi_{\Delta, \bar{\Delta}}(z, \bar{z})$ and a nonzero central charge $c$ appears.

The dynamics of a conformal field theory are defined by the transformation properties of its physical primary fields under the $L_{n}$ and $\bar{L}_{n}$ Virasoro algebras. The primary fields transform under $(15)$ as $\phi_{\Delta, \bar{\Delta}}^{\prime}(z, \bar{z})=\left(\frac{\partial z^{\prime}}{\partial z}\right)^{\Delta}\left(\frac{\partial \bar{z}^{\prime}}{\partial \bar{z}}\right)^{\bar{\Delta}} \phi_{\Delta, \bar{\Delta}}\left(z^{\prime}, \bar{z}^{\prime}\right)$. Thus, their commutators with the $L_{n}$ are:

$$
\left[L_{n}, \phi_{\Delta, \bar{\Delta}}(z, \bar{z})\right]=z^{m+1} \partial_{z} \phi_{\Delta, \bar{\Delta}}(z, \bar{z})+\Delta(m+1) z^{m} \phi_{\Delta, \bar{\Delta}}(z, \bar{z})
$$

Similar relations hold for $\bar{L}_{n}$ commutators. The central charge and the numbers $(\Delta, \bar{\Delta})$, which define the representations by the primary fields $\phi_{\Delta, \bar{\Delta}}(z, \bar{z})$, characterize the conformal field theory. The primary fields form highest weight representations of these algebras.

$$
\begin{array}{ll}
n>0 & L_{n} \phi_{\Delta, \bar{\Delta}}(z, \bar{z})\left|0>=0, L_{0} \phi_{\Delta, \bar{\Delta}}(z, \bar{z})\right| 0>=\Delta \phi_{\Delta, \bar{\Delta}}(z, \bar{z}) \mid 0> \\
n>0 & \bar{L}_{n} \phi_{\Delta, \bar{\Delta}}(z, \bar{z})\left|0>=0, \bar{L}_{0} \phi_{\Delta, \bar{\Delta}}(z, \bar{z})\right| 0>=\bar{\Delta} \phi_{\Delta, \bar{\Delta}}(z, \bar{z}) \mid 0>
\end{array}
$$

$\Delta$ and $\bar{\Delta}$ are independent real eigenvalues of $L_{0}$ and $\bar{L}_{0}$ respectively. The physical states 8 are created by the action of $L_{-|n|}$ and $\bar{L}_{-|m|}$ on $\mid 0>$. The states have the following form:

$$
\phi_{\Delta, \bar{\Delta}}^{[\bar{n}, \bar{m}]}\left|0>=L_{-\left|n_{1}\right|} \ldots L_{-\left|n_{k}\right|} \ldots \bar{L}_{-\left|m_{1}\right|} \ldots \bar{L}_{-\left|m_{p}\right|} \phi_{\Delta, \bar{\Delta}}\right| 0>
$$

Physical considerations and unitarity, constrain the central charge and the dimensions $(\Delta, \bar{\Delta})$ to special rational values ${ }^{9}[8][9]$. The limitation on allowed dimensions leads to a fundamentally important result. The space of states over each primary field contains nontrivial null vectors [12]. The simplest example is the state:

$$
\left[L_{-2}+\frac{3}{2(2 \Delta+1)}\left(L_{-1}\right)^{2}\right] \phi_{\Delta, \bar{\Delta}} \mid 0>=0
$$

From (16), (18) and the proper conjugation on the $L_{n}$ 's $\left(L_{n}^{\dagger}=L_{-n}\right)$, the reader can easily show that this state has zero norm for $\Delta=\frac{1}{16}[5-c \pm \sqrt{(1-c)(25-c)}]$. There are different null vectors in the space of states above every primary field. V. Kac has classified all the null vectors [12].

The null vectors lead to differential equations that are crucial to the construction of the correlation functions. The derivation of these equations requires a detailed knowledge

${ }^{8} \mid 0>$, the ground state, is also a highest weight state, i.e. $L_{|n|}\left|0>=\bar{L}_{|n|}\right| 0>=0$. Invariance of $\mid 0>$ under the finite conformal group already required: $L_{n}\left|0>=\bar{L}_{n}\right| 0>=0$ for $n=-1,0,1$. See equation (12) and accompanying text.

${ }^{9}$ For $0<c<1$ the result is: $c=1-\frac{6}{m(m+1)}, \Delta_{r s}=\frac{[r(m+1)-s m]^{2}-1}{4 m(m+1)}$ with $1 \leq r \leq m-1$, $1 \leq s \leq r$ and $m=2,3,4, \ldots$ 
of field theory. A simple understanding of their origin can be obtained if one recalls that any coordinate transformation on fields can be generated through the action of the energymomentum tensor (Noether's Theorem). This includes the conformal transformations of (15). Thus, the energy-momentum tensor must be related to the $L_{n}$ 's and the $\bar{L}_{n}$ 's that generate the two Virasoro algebras. If the theory is conformally invariant, one can show that these operators are the Laurent expansion coefficients of the energy-momentum tensor. One can use this identification and the explicit form of conformal transformations on primary fields (see below (16)) to rewrite the action of the $L_{-n}$ and $\bar{L}_{-n}$ 's with differential operators [8]. This involves a long calculation using operator product results (17)-(18). One finally obtains the following equation for a correlation function with $P$ primary fields $\phi_{\Delta_{j}, \bar{\Delta}_{j}}\left(z_{j}, \bar{z}_{j}\right), j=1, \ldots, P$ and $L_{-n} \phi_{\Delta, \bar{\Delta}}(z, \bar{z})$ :

$$
\begin{array}{r}
<<\left(L_{-n} \phi_{\Delta, \bar{\Delta}}(z, \bar{z})\right) \phi_{\Delta_{1}, \bar{\Delta}_{1}}\left(z_{1}, \bar{z}_{1}\right), \ldots, \phi_{\Delta_{P}, \bar{\Delta}_{P}}\left(z_{P}, \bar{z}_{P}\right)>>=(-1)^{n-1} \times \\
\sum_{j=1}^{P}\left(\frac{(1+n) \Delta_{j}}{\left(z-z_{j}\right)^{n}}-\frac{1}{\left(z-z_{j}\right)^{n-1}} \frac{\partial}{\partial z_{j}}\right) \begin{array}{r}
<\phi_{\Delta, \bar{\Delta}}(z, \bar{z}) \phi_{\Delta_{1}, \bar{\Delta}_{1}}\left(z_{1}, \bar{z}_{1}\right), \ldots \\
\ldots, \phi_{\Delta_{P}, \bar{\Delta}_{P}}\left(z_{P}, \bar{z}_{P}\right)>>
\end{array}
\end{array}
$$

If we substitute these differential operator forms in null vector equations, like (20), we obtain homogeneous equations. The resulting equations are extremely powerful. Since the correlation functions solve these equations, they must be linear combinations of the general solutions. The independent solutions that are analytic in the $z_{j}$ are called conformal blocks. For the rational models there are only a finite number, and they transform under the braid group. The form of the conformal blocks are known for all rational conformal theories [10].

The Ising model affords us with a simple example. It has central charge $c=1 / 2$. The local energy density, $E(z, \bar{z})$, has conformal dimensions $(\Delta, \bar{\Delta})=(1 / 2,1 / 2)$. Thus, correlations involving this field satisfy the equation:

$$
\begin{array}{r}
0=\sum_{j=1}^{P}\left(\frac{\Delta_{j}}{\left(z-z_{j}\right)^{2}}+\frac{1}{\left(z-z_{j}\right)} \frac{\partial}{\partial z_{j}}-\frac{3}{4} \partial_{z}^{2}\right)<<E(z, \bar{z}) \phi_{\Delta_{1}, \bar{\Delta}_{1}}\left(z_{1}, \bar{z}_{1}\right), \ldots \\
\ldots, \phi_{\Delta_{P}, \bar{\Delta}_{P}}\left(z_{P}, \bar{z}_{P}\right)>>
\end{array}
$$

We write the two independent analytic conformal blocks for correlations involving four $E\left(z_{j}, \bar{z}_{j}\right)$.

$$
\frac{[x(1-x)]^{2 / 3}}{\left(z_{1}-z_{3}\right)\left(z_{2}-z_{4}\right)} F(4 / 3,3,8 / 3 ; x) \text { and } \frac{F(-2,-1 / 3,-2 / 3 ; x)}{[x(1-x)]\left(z_{1}-z_{3}\right)\left(z_{2}-z_{4}\right)}
$$

The functions $F(a, b, c, x)$ are hyper-geometric functions and $x \equiv \frac{\left(z_{1}-z_{2}\right)\left(z_{3}-z_{4}\right)}{\left(z_{1}-z_{3}\right)\left(z_{2}-z_{4}\right)}$. They are analytic functions with cuts for $x \sim 0$. Using integral representations for $F(a, b, c, x)$, one can show that they transform nontrivially under the $\sigma$-braids of (5). For example, $F(-2,-1 / 3,-2 / 3 ; x)=1-x+x^{2}$. Thus, the braid transformation that exchanges $z_{3}$ with $z_{2}$, e.g. $x \longrightarrow x^{-1}$, simply multiplies the second block by $(-1)$. 
We can write the correlation function itself. In this case, it is the absolute square of the second block of (23):

$$
<<E\left(z_{1}, \bar{z}_{1}\right) \ldots E\left(z_{4}, \bar{z}_{4}\right)>>=\left(\frac{1-x+x^{2}}{z_{13} z_{24}(1-x) x}\right)\left(\frac{1-\bar{x}+\bar{x}^{2}}{\bar{z}_{13} \bar{z}_{24}(1-\bar{x}) \bar{x}}\right)
$$

This correlation function is easily seen to be invariant under braid group transformations. This is the generic situation. The correlation functions are invariant under braiding, but the conformal blocks transform in nontrivial and often multi-dimensional representations (see below). The null vector equations, (20) to (22) reduce the construction of correlation functions to a problem in constructing invariants of the braid group.

Braid statistics enters conformal field theories through the concept of factorization. This can already be seen in the 3-point correlations, (14), when written with complex coordinates:

$$
\begin{gathered}
<<\phi_{i}\left(z_{1}, \bar{z}_{1}\right) \phi_{j}\left(z_{2}, \bar{z}_{2}\right) \phi_{k}\left(z_{3}, \bar{z}_{3}\right)>>=C_{i j k} W_{k}^{i j}\left(z_{1}, z_{2}, z_{3}\right) \bar{W}_{k}^{i j}\left(\bar{z}_{1}, \bar{z}_{2}, \bar{z}_{3}\right) \\
W_{k}^{i j}\left(z_{1}, z_{2}, z_{3}\right)=\frac{1}{\left(z_{1}-z_{2}\right)^{\Delta_{i}+\Delta_{j}-\Delta_{k}} \times \text { cyclic perm }}
\end{gathered}
$$

The $W_{k}^{i j}$ 's are analytic in the coordinates and transform as one dimensional representations of the braid group. For example, the $\sigma$ - braid that twists $z_{1}$ around $z_{2}$ in a clockwise sense gives:

$$
\sigma_{1} W_{k}^{i j}\left(z_{1}, z_{2}, z_{3}\right)=e^{i \pi\left(\Delta_{i}+\Delta_{j}-\Delta_{k}\right)} W_{k}^{j i}\left(z_{2}, z_{1}, z_{3}\right)
$$

A partial factorization also occurs for all higher correlation functions.

$$
\begin{aligned}
<<\phi_{j_{1}}\left(z_{1}, \bar{z}_{1}\right) \ldots \phi_{j_{P}}\left(z_{P}, \bar{z}_{P}\right)>> & \\
& =\sum_{k, l} A_{k l} F_{l}^{j_{1} \ldots j_{P}}\left(z_{1}, \ldots, z_{P}\right) \bar{F}_{k}^{j_{1} \ldots j_{P}}\left(\bar{z}_{1}, \ldots, \bar{z}_{P}\right)
\end{aligned}
$$

The sum over conformal blocks $F_{l}^{j_{1} \ldots j_{P}}$ is labeled by the generic indices $k$ and $l$. The blocks are the complete set of analytic solutions to the null vector equations, i.e. (20)-(22). Since there are only a finite number, the sums are finite. The transformation properties of the conformal blocks under the braid group can be seen by writing them as Coulomb gas contour integrals [10].

The remaining constants, $A_{l k}$, can be determined by imposing the commutativity ${ }^{10}$ and associativity of products of fields on the forms in (27). The associativity comes from a completeness relation. The product of any two fields can be expressed as a sum over all primary fields and their conformal descendants (19).

$$
\phi_{i}\left(z_{1}, \bar{z}_{1}\right) \phi_{j}\left(z_{2}, \bar{z}_{2}\right)=\sum_{k, \vec{n}, \vec{m}} C_{i j k} \beta_{k, n}^{i j}\left(z_{1}, z_{2}\right) \bar{\beta}_{k, m}^{i j}\left(\bar{z}_{1}, \bar{z}_{2}\right) \phi_{\Delta_{k}, \bar{\Delta}_{k}}^{(\vec{n}, \vec{m})}\left(z_{2}, \bar{z}_{2}\right)
$$

Here, the constants $C_{i j k}$ are the structure constants from (14), and $\beta_{k}^{i j}$ is an analytic function of its arguments that can be determined from (17) and (18). In the following, it is sufficient to consider the 4 -point correlations at the special points: $z_{1}=0, z_{2}=x, z_{3}=1$,

\footnotetext{
${ }^{10}$ This results from the fact that all products of fields are actually time ordered, i.e. $\left(\phi_{1} \phi_{2}\right)_{+}=$ $\left(\phi_{2} \phi_{1}\right)_{+}$.
} 
and $z_{4}=\infty$. Then, the blocks only depend on the $z_{j}$ through $x$. The correlation function at arbitrary values of $z_{1}, \ldots, z_{4}$ can be related to correlations at these special values through the invariances of the finite conformal group of (12), i.e. $L_{k}\left|0>=\bar{L}_{k}\right| 0>=0$ for $k=-1,0,1$. Applying (27) and (13) to the 4-point correlations, we find that:

$$
\begin{aligned}
G_{m n}^{l k}(x, \bar{x}) & =\sum_{p} C_{n k p} C_{l m p} F_{l m}^{n k}(p \mid x) \bar{F}_{l m}^{n k}(p \mid \bar{x}) \\
& =\sum_{q} C_{k l q} C_{n m q} F_{n m}^{k l}(q \mid 1-x) \bar{F}_{n m}^{k l}(q \mid 1-\bar{x}) \\
G_{m n}^{l k}(x, \bar{x}) & =<<\phi_{n}(0,0) \phi_{k}(x, \bar{x}) \phi_{l}(1,1) \phi_{m}(\infty, \infty)>>
\end{aligned}
$$

Here, the $F_{l m}^{n k}$ 's are the conformal blocks. In the first equality, we applied the operator product expansion (28) to the products $\left(\phi_{n} \phi_{k}\right)$ and $\left(\phi_{l} \phi_{m}\right)$ and then calculated the remaining 2-point correlation with (13). In the second equality, we applied (28) to the products $\left(\phi_{k} \phi_{l}\right)$ and $\left(\phi_{n} \phi_{m}\right)$ and then used (13). The equality (29) is known as the duality relation [8]. It is extremely powerful. It provides an inhomogeneous algebraic equation for the structure constants $C_{i j k}$ that has enabled their explicit calculation [10].

Now, we can show that this equation is also related to braiding. Since the blocks $F_{l m}^{n k}(p \mid x)$ are independent for different values of $\mathrm{p}$, the duality relationship leads to a separate equation for each value of $\mathrm{p}$. The conformal blocks as a function of $x$ can be rewritten in terms of the complete set of blocks as a function of $1-x$. This generates matrix equations that also have a graphical interpretation, fig. 2.

$$
F_{l m}^{n k}(p \mid x)=\sum_{q} T\left(p, q,\left[\begin{array}{l}
k l \\
n m
\end{array}\right]\right) F_{n m}^{k l}(q \mid 1-x)
$$

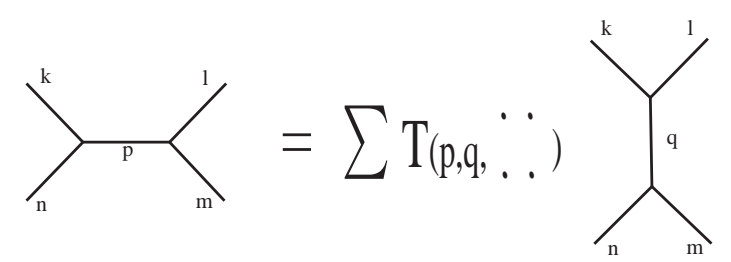

Fig. 2. Duality relation for a single conformal block. Here, $p$ and $q$ index primary fields.

By the commutativity of the primary fields, the correlation function is invariant under the exchange of any two fields, i.e. invariant under any element of the braid group (5).

$$
<<\ldots \phi_{i} \ldots \phi_{j} \ldots>>=<<\ldots \phi_{j} \ldots \phi_{i} \ldots>>
$$

Nevertheless, a glance at the example in (23) will show that the conformal blocks form nontrivial representations of the braid group. Thus, invariance of correlations under braid transformations is only possible if conformal blocks transform linearly under the braid group. This leads to a second diagrammatic relationship, fig. 3, [13]:

$$
F_{l m}^{n k}(p \mid x)=\sum_{q} B\left(p, q,\left[\begin{array}{l}
n k \\
l m
\end{array}\right]\right) F_{k m}^{n l}(q \mid \ldots)
$$




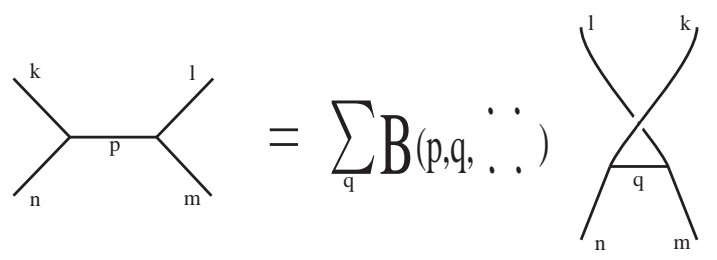

Fig. 3. $\sigma_{k l}$-braid relation on conformal blocks

The braiding matrices $B\left(p, q,\left[\begin{array}{l}n k \\ l m\end{array}\right]\right)$ have interesting properties. For example, (32) implies that they must satisfy nontrivial commutation relations with the structure constants, see (29). Finally, through the series of braid transformations shown in fig. 4, one can show that the duality matrix $T\left(p, q,\left[\begin{array}{c}k l \\ n m\end{array}\right]\right)$, and the braiding matrix $B\left(p, q,\left[\begin{array}{l}n k \\ l m\end{array}\right]\right)$ are linearly related.

$$
B\left(p, q,\left[\begin{array}{l}
n k \\
l m
\end{array}\right]\right)=e^{-i \pi\left[\Delta_{p}+\Delta_{q}-\Delta_{l}-\Delta_{m}\right]} T\left(p, q,\left[\begin{array}{l}
n m \\
l k
\end{array}\right]\right)
$$
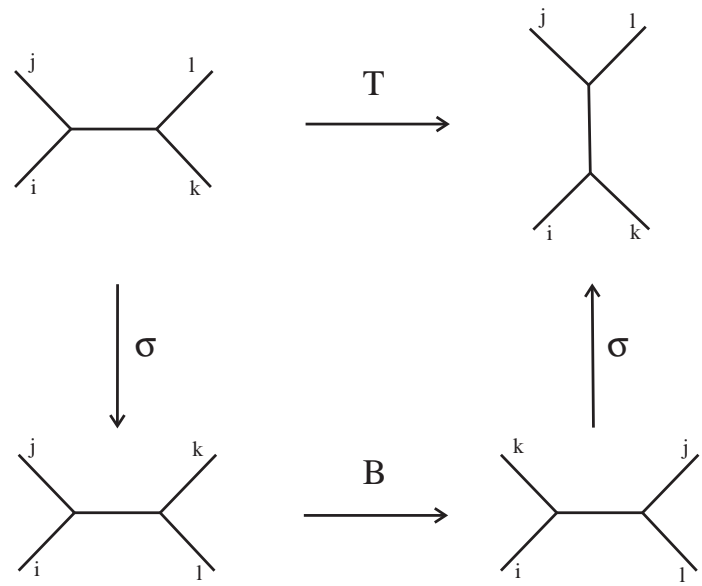

Fig. 4. Proof of the relation between the braiding and duality matrices

Here, we have also used the braid transformations of the 3 -point vertex (26). Thus, the braid and duality properties of the conformal blocks are intimately related [8, 13, 14]. As we have already stated, the duality properties have been the basis of extensive calculations of the structure constants $[10,8]$ The relation between the braiding and duality matrices says that solving the duality equation is equivalent to requiring that correlation functions be braid invariants.

The braid transformations of the conformal blocks were extensively developed in [13]. It may not surprise the present audience that those authors have shown deep relationships with quantum groups. Other authors have furthered the ideas of [13] and have used the conformal blocks of rational conformal models to construct knot invariants [14]. That application is well beyond the scope of this review and the present authors' activities. Nevertheless, the reviews on those applications [14] should be accessible to a reader who has followed this presentation to the end. 


\section{References}

[1] J. M. Leinaas and J. Myhreim, Il Nuovo Cimento 37B (1977) 1.

[2] Y.-S. Wu, Phys. Rev. Lett. 52 (1984) 2103; J. Birman, Links, Braids and Mapping Class Groups, (Princeton University Press 1974).

[3] D. P. Arovas, R. Schrieffer, F. Wilczek, and A. Zee, Nucl. Phys. B251 (1985) 117.

[4] for a review see: F. Wilczek, Fractional Statistics and Anyon Superconductivity, (World Scientific 1990); A. Comtet, J. McCabe, and S. Ouvry, Images de la Physique, (1992) 21.

[5] see generally: M. Sporre, J. J. Verbaarshot, and I. Zahed, Phys. Rev. Lett. 67 (1991) 1817; G. V. Dunne, A. Lerda, and C. A. Trugenberger, Int. J. of Mod. Phys. B5 (1991) 1675; ibid Nucl. Phys. B370 (1992) 601; A. Khare, J. McCabe, and S. Ouvry, Phys. Rev. D46 (1992) 2714.

[6] R. B. Laughlin, Phys. Rev. Lett. 60 (1988) 2677; Y.-H. Chen, F. Wilczek, E. Witten, and B. I. Halperin, Int. J. of Mod. Phys. B3 (1989) 1001.

[7] A. M. Polyakov, JETP Lett. 12 (1970) 381.

[8] A. A. Belavin, A. M. Polyakov, and A. B. Zamolodchikov, Nucl. Phys. B241 (1984) 333; for a review see articles of P. Ginsparg and J. Cardy in Les Houches Summer School, 1988 (Eds. E. Brezin and J. Zinn-Justin).

[9] D. Friedan, Z. Qiu, and S. Shenker, Phys. Rev. Lett. 52 (1984) 1575.

[10] Vl. S. Dotsenko and V. Fateev, Nucl. Phys. B240 (1984) 312; ibid, B251 (1985) 691; Vl. Dotsenko, Nucl. Phys. B235 (1984) 54; J. McCabe and T. Wydro, ENSLAPP-L-526/95 (cond-mat/9507033).

[11] G. Von Gehlen, V. Rittenberg, and T. Vescan, J. of Physics A20 (1987) 2577; G. T. Barkema and J. McCabe, J. Stat. Phys. 84 (1996) 1067.

[12] V. Kac, Lecture Notes in Physics 94, (Springer 1979).

[13] G. Moore and N. Seiberg, Phys. Lett. 212B (1988) 451; also in Superstrings 1989, (Eds. M. Green, R. Iengo, S. Randjbar-Daemi, E. Sezgin, and A. Strominger) 1.

[14] L. Alvarez-Gaume, C. Gomez, and G. Sierra, in Physics and Mathematics of Strings, (Eds. L. Brink, D. Friedan, and A.M. Polyakov) 16. 